\title{
Temporal profile monitor based on electro-optic spatial decoding for low-energy bunches
}

\author{
Wei Wang, Yingchao Du, Lixin Yan, Zhijun Chi, Zhen Zhang, \\ Jianfei Hua, Wenhui Huang, and Chuanxiang Tang \\ Department of Engineering Physics, Tsinghua University, Beijing 100084, China \\ Ming Li \\ Institute of Applied Electronics, China Academy of Engineering Physics, Mianyang 621900, China
}

(Received 21 June 2017; published 3 November 2017)

\begin{abstract}
The measurement of electron bunch temporal profile is one of the key diagnostics in accelerators, especially for ultrashort bunches. The electro-optic (EO) technique enables the precise longitudinal characterization of bunch electric field in a single-shot and nondestructive way, which can simultaneously obtain and analyze the time jitter between the electron bunch and the synchronized laser. An EO monitor based on spatial decoding for temporal profile measurement and timing jitter recoding has recently been demonstrated and analyzed in depth for low-energy bunches at the Tsinghua Thomson scattering X-ray source. A detailed description of the experimental setup and measurement results are presented in this paper. An EO signal as short as $82 \mathrm{fs}$ (rms) is observed with $100 \mu \mathrm{m}$ gallium phosphide for a $40 \mathrm{MeV}$ electron bunch, and the corresponding length is $106 \mathrm{fs}(\mathrm{rms})$ with $300 \mu \mathrm{m}$ zinc telluride. Owing to the field-opening angle, we propose a method to eliminate the influence of energy factor for bunches with low energy, resulting in a bunch length of $\sim 60 \mathrm{fs}$ (rms). The monitor is also successfully applied to measure time jitter with approximately $10 \mathrm{fs}$ accuracy. The experiment environment is proved to be the main source of the slow drift, which is removed using feedback control. Consequently, the rms time jitter decreases from 430 fs to $320 \mathrm{fs}$.
\end{abstract}

DOI: 10.1103/PhysRevAccelBeams.20.112801

\section{INTRODUCTION}

Ultrashort relativistic electron bunches at a duration of $100 \mathrm{fs}$ or less are highly important in many accelerator facilities, especially in x-ray free electron lasers [1-3]. Precise measurements of temporal profile for such bunches have been proven to be essential and complicated. A nondestructive diagnostic technique, which can realize online measurements of longitudinal bunch profile in a single-shot way, is always preferred. The electro-optic (EO) detection techniques meet these conditions because of its noninvasive setup, which can simultaneously work as a real-time monitor during normal operation of the facilities for customers and other experiments.

Pump-probe techniques are usually used to study ultrafast physical processes in the subpicosecond regime wherein the sample is pumped by a high energy density laser and then probed by an ultrafast electron bunch within a time delay. The time jitter between pump laser and probe electron will cause a reduction of temporal resolution $[4,5]$.

\footnotetext{
*huangwh@mail.tsinghua.edu.cn

Published by the American Physical Society under the terms of the Creative Commons Attribution 4.0 International license. Further distribution of this work must maintain attribution to the author(s) and the published article's title, journal citation, and DOI.
}

The EO detection is able to measure the jitter for each pump-probe event by recording the arrival time of each individual electron bunch with respect to the laser pulse $[6,7]$. According to the arrival time jitter obtained in the EO detection, slow drift can be removed online and postprocessing (sorting) of the data is also possible for pumpprobe experiments. Therefore, the EO detection can serve as a potential tool to improve the time resolution in the pump-probe experiment and reduce photon yield jitter at Thomson scattering X-ray source.

The single-shot EO measurement was first performed in 2002 [8], and different variants occurred later [9-16]. All the EO techniques share the same principle of detecting the field-induced birefringence in the EO crystal caused by the local Coulomb electric field of the electron beam. The Coulomb field for a highly relativistic electron is intensely concentrated into the plane perpendicular to the travel direction. With the superposition of field for each electron, the Coulomb field of the bunch with high energy almost equals the longitudinal density distribution. Considering the field-opening angle in the low-energy region, the field profile measured in EO methods results in length broadening compared with the bunch profile associated with energy factor and distance between probe laser and electron bunch. Except for directly measuring the Coulomb electric field, indirectly characterizing the terahertz $(\mathrm{THz})$ field of bunch radiation is also an alternative way $[17,18]$. 
Spatial decoding EO detection was first demonstrated in 2005 [10]. The approach converts bunch temporal profile to spatial intensity modulation of the probe laser through sweeping the ultrashort laser across an EO crystal at an angle to the bunch travel direction. Spatial decoding technique as a nondestructive method reaches a high temporal resolution generally limited by the frequency response of EO crystal, typically zinc telluride (ZnTe) and gallium phosphide $(\mathrm{GaP})$. Besides, this technique may be appropriate to measure beam arrival time because of its relatively simple setup of signal detection $[6,10]$.

In the present paper, we applied this previously developed technique to measure the longitudinal properties of the electrons at Tsinghua Thomson scattering X-ray source (TTX), and made some improvements. Owing to the oblique incidence of the laser through the crystal, previously measured results by spatial decoding EO detection are not the real bunch distribution, especially for long bunches. We remove the influence of the oblique incidence to determine the real bunch shape in Sec. IV B. Then, we take advantage of the variable distance to eliminate the field broadening for low-energy bunches in Sec. IV E. At last, this technique is applied in a feedback mode to reduce bunch arrival time jitter in Sec. IV G.

\section{PRINCIPLE DESCRIPTION}

The electric field of a relativistic electron can be obtained by Lorentz transformation to electrostatic field of resting point charge [19] illustrated as follows:

$$
\boldsymbol{E}_{0}=\frac{q}{4 \pi \epsilon_{0} r^{2}} \frac{1-\beta^{2}}{\left(1-\beta^{2} \sin ^{2} \theta\right)^{3 / 2}} \boldsymbol{e}_{r},
$$

where $q=-e$ is the electron charge, $\epsilon_{0}$ is the vacuum permittivity, $r$ is the distance to the charge, $\beta=\nu / c$ is the charge speed, $\boldsymbol{e}_{r}$ is the unit radius vector from the electron to the observer, and $\theta$ is the angle between $\boldsymbol{e}_{r}$ and the travel direction of the electron. Unlike the electron at rest, the field of a relativistic one is concentrated into the plane perpendicular to the moving direction and forms a flat disc with an opening angle of $2 / \gamma$ [19], which is defined as field-opening angle in this paper. The Coulomb field of an electron bunch is the convolution of longitudinal charge distribution $\rho(t)$ and electric field of a single electron illustrated as follows:

$$
\boldsymbol{E}=\boldsymbol{E}_{0} * \rho(t)
$$

For highly relativistic bunches, the longitudinal field component is much smaller than the radial component and can be neglected. The radial field becomes [20]

$$
E_{r}(r, t)=\frac{\rho(t)}{2 \pi \epsilon_{0} r},
$$

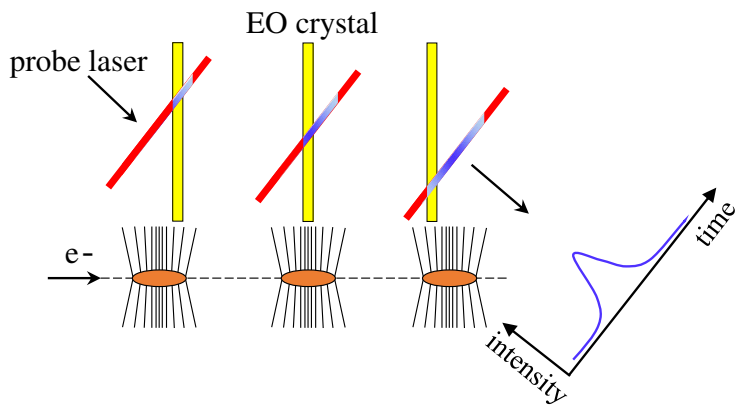

FIG. 1. Schematic description of spatial decoding EO detection (top view).

and is almost identical to the longitudinal density distribution except for a slight temporal broadening because of the opening angle.

When a relativistic electron bunch passes through a nearby EO crystal, the Coulomb field of the bunch will induce birefringence in the crystal, which would change the polarization direction of the laser simultaneously transmitting through the crystal. In the spatial decoding EO detection seen in Fig. 1, an ultrashort probe laser transfers across the crystal with an angle to the beam travel direction. Thus, the Coulomb field of different bunch slices modulates the corresponding section in the transverse profile of laser pulse. The temporal profile of the bunch is mapped to the space distribution of the laser and can be detected by a charge-couple device (CCD) camera.

Although the oblique incidence would cause the complexity of data corrections, there still exists some distinct advantages in spatial decoding EO detection. First, the bunch shape is mapped into the laser transverse profile instead of the temporal profile in the normal incidence techniques. Therefore, the EO signal can be directly detected by a CCD camera, which is much simpler than a spectrometer [8] or cross-correlation [9]. Meanwhile, the resolution of arrival time is much better for the simplicity of signal detection than the normal incidence techniques. On the other hand, the distance of the electron path to the interaction part of laser beam can be easily changed by adjusting the laser delay, which is fixed in the normal incidence techniques. Measuring bunch length at different distance creates the possibility to eliminate the field broadening for low-energy bunches and obtain results shorter than the response limitations of the EO crystal.

The relative phase retardation of the probe laser caused by the field-induced birefringence is [21]

$$
\Gamma=\frac{2 \pi}{\lambda}\left(n_{1}-n_{2}\right) d=\frac{2 \pi d}{\lambda} n_{0}^{3} r_{41} E_{r},
$$

assuming the setup of maximum EO effect where $d$ is the crystal thickness, $\lambda$ is the wavelength of the probe laser, $n_{0}$ is the refractive index of the crystal at wavelength $\lambda$, and $r_{41}$ is the EO coefficient of the crystal. In the crossed 


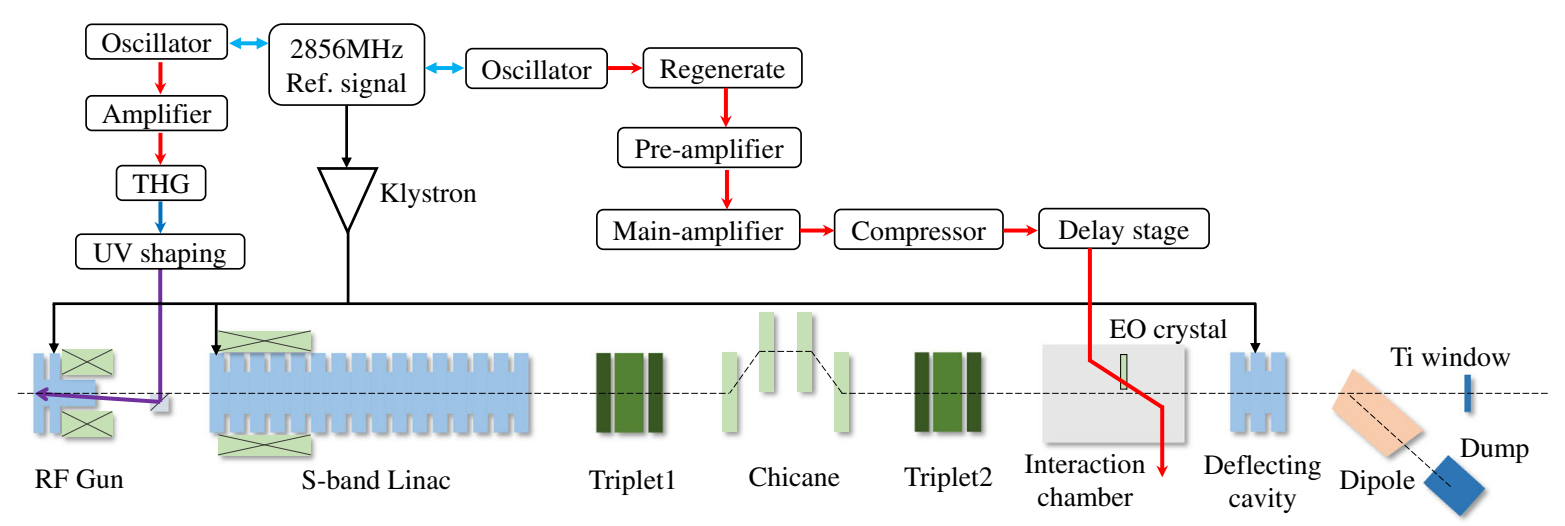

FIG. 2. Schematic layout of the TTX beam line and laser system.

polarization setup where the vertically polarized component is detected after a horizontally polarized probe laser $I_{\text {in }}$ passing through the EO crystal, the laser intensity of signal $I_{\text {det }}$ is given by [22]

$$
I_{\mathrm{det}}=I_{\mathrm{in}} \sin ^{2}\left(\frac{\Gamma}{2}\right)=I_{\mathrm{in}} \sin ^{2}\left(\frac{\pi d}{\lambda} n_{0}^{3} r_{41} E_{r}\right)
$$

For phase shift $\Gamma \ll 1$, the intensity is proportional to the square of bunch Coulomb field.

\section{EXPERIMENT SETUP}

This detection was successfully demonstrated at TTX $[23,24]$. A schematic drawing of the beam line and laser system is illustrated in Fig. 2. The electron bunches are generated in a modified version of the BNL/KEK/SHI type 1.6 cell photocathode radio-frequency (rf) gun and are rapidly accelerated to 4-5 MeV. A 3 m SLAC-type traveling wave accelerating section increases the bunch energy to $40-50 \mathrm{MeV}$ at a charge of $\sim 1 \mathrm{pC}$ to $\sim 1 \mathrm{nC}$ at a repetition rate of $10 \mathrm{~Hz}$. A magnetic chicane is used to longitudinally compress the bunch from $\sim 10$ ps to $100 \mathrm{fs}$ by introducing an energy slope through the bunch by offcrest acceleration in the accelerating section. A deflecting cavity is used to measure the longitudinal profile of the bunch as a comparison with the EO detection. A $50 \mathrm{MW}$ TOSHIBA klystron provides the entire power for the gun, accelerating section, and deflecting cavity.

A Ti:sapphire laser system from Coherent Inc. was used to generate the ultraviolet (UV) driving laser for the photocathode $\mathrm{rf}$ gun, which can provide $\sim 1.5 \mathrm{~mJ}$ UV laser with $0.3-10$ ps pulse length at a central wavelength of $266 \mathrm{~nm}$. The $30 \mathrm{TW}$ ultrashort infrared (IR) scattering laser is delivered by the second Ti:sapphire laser system from Amplitude Technologies. The pulse energy is $\sim 0.7 \mathrm{~J}$ at the interaction point with $30 \mathrm{fs}$ (FWHM) pulse length at $800 \mathrm{~nm}$ central wavelength. The probe laser for the EO experiment is split from the scattering laser at a pulse energy of $100 \mu \mathrm{J}$. Operating at a repetition frequency of
79.3 MHz, both Ti:sapphire oscillators are phase-locked to the $2856 \mathrm{MHz}$ master frequency of the accelerator. Both amplifiers generate UV and IR pulses at a repetition rate of $10 \mathrm{~Hz}$. A detailed description of the beam line and laser system can be found in Refs. $[25,26]$.

The experimental setup for EO detection in the vacuum chamber is shown in Fig. 3. After passing through a compressor, the horizontally polarized probe laser is shaped to a strip by a set of two cylindrical lenses $\left(f_{1}=50 \mathrm{~cm}\right.$, $f_{2}=8 \mathrm{~cm}$ ). To decrease pulse broadening because of nonlinear group dispersion, the total thickness of the two lenses is less than $5 \mathrm{~mm}$. As one of the limiting factors to temporal resolution, the pulse length of probe laser delivered to the EO crystal is $40 \mathrm{fs}$ (FWHM) measured by an autocorrelator. The probe laser is injected into the EO crystal at an angle of $40^{\circ}$ relative to the normal of the crystal surface. The transverse profile of the strip laser is $1 \mathrm{~mm}$ in width and $5 \mathrm{~mm}$ effective length on the crystal, which covers a time window of 14 ps.

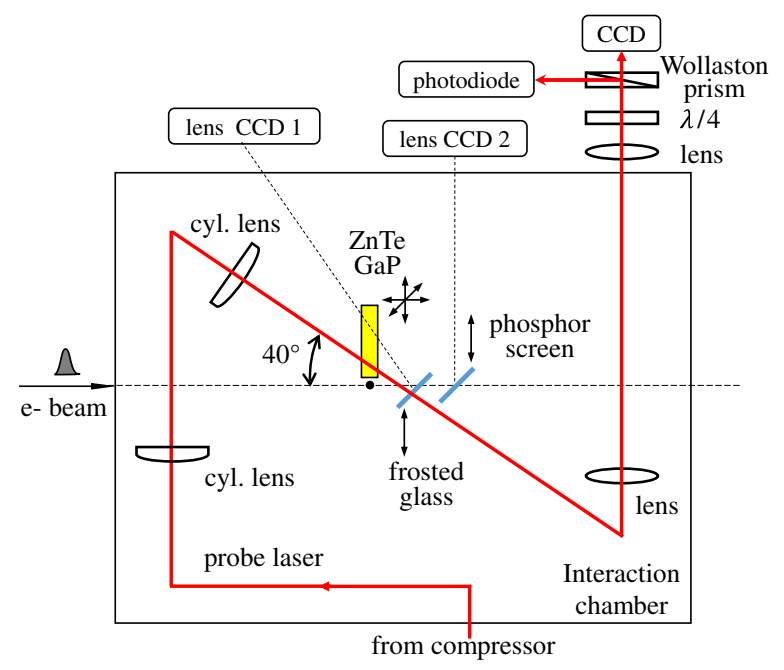

FIG. 3. Experimental setup in vacuum interaction chamber and laser transmission path for the spatial decoding EO detection (top view). 
Two kinds of plane parallel EO crystals are used for the measurements, namely, $300 \mu \mathrm{m}$ ZnTe (MaTecK, Juelich) and $100 \mu \mathrm{m} \mathrm{GaP}$ (Moltech, Berlin) with a size of $10 \times 10 \mathrm{~mm}^{2}$. Both ZnTe and GaP are cut in the (110) plane, and the direction of [-110] axis is along one marked edge. The crystals are mounted to make sure that the $[-110]$ axis is in the horizontal direction and points the center of the beam pipe. In our setup for EO detection, the electron beam and probe laser are on the same level. Therefore, the angle of the electric field direction is changeless, and the signal dependence on the angle is ignored. Both crystals are mounted on a 3-dimension movable stage, which can choose the kind of detection crystal and change the crystal position without opening the vacuum chamber. The typical distance from electron beam to the crystal edge is $0.5 \mathrm{~mm}$ in the signal acquisition progress.

Modulated within the crystal by the beam Coulomb field, the laser pulse is transferred to the crossed polarization setup by a pair of lenses $(f=30 \mathrm{~cm})$ through image relaying. The quarter wave plate is used to compensate the residual birefringence of the EO crystal caused by imperfections and mechanical stress. The horizontally polarized component of the laser pulse is detected by a fast photodiode, together with the raw signal of a stripe beam position monitor to identify the time overlap of laser pulse and electron bunch at 500 ps accuracy. This time window is then swept by adjusting the delay stage in small steps to find the EO signal. A phosphor screen and a frosted glass are mounted on two motored stages and observed by two CCD cameras, respectively. They are used to confirm the bunch transverse profile and ensure the stability of the laser in air or in vacuum. The frosted glass is also used to observe the relative position of the laser and electron to make sure that they are on the same level.

\section{RESULT AND DISCUSSION}

\section{A. Signal acquisition and time calibration}

The measurement was performed at crossed polarization and the typically measured EO signal by single-shot spatial decoding EO detection is shown in Fig. 4. The bunch is compressed by the chicane for a short length at a bunch charge of $300 \mathrm{pC}$ and energy of $40 \mathrm{MeV}$. To avoid additional noise introduced by background subtraction because of the laser instabilities, the laser background without electron beam is an average of 500 shots taken with the same laser and CCD settings. The typically measured amplitude of phase retardation is $\Gamma_{\max }=0.12$ for $300 \mu \mathrm{m}$ $\mathrm{ZnTe}$, which is approximately 7.7 times the magnitude of $100 \mu \mathrm{m} \mathrm{GaP}$. Therefore, the phase retardation is small enough to assume $\sin ^{2}(\Gamma / 2) \approx(\Gamma / 2)^{2}$. According to Eq. (5), the raw EO signal (laser background subtracted) is proportional to the square of the Coulomb field. Figure 4 is the raw data of EO detection and the $E_{r}^{2}$ dependence is
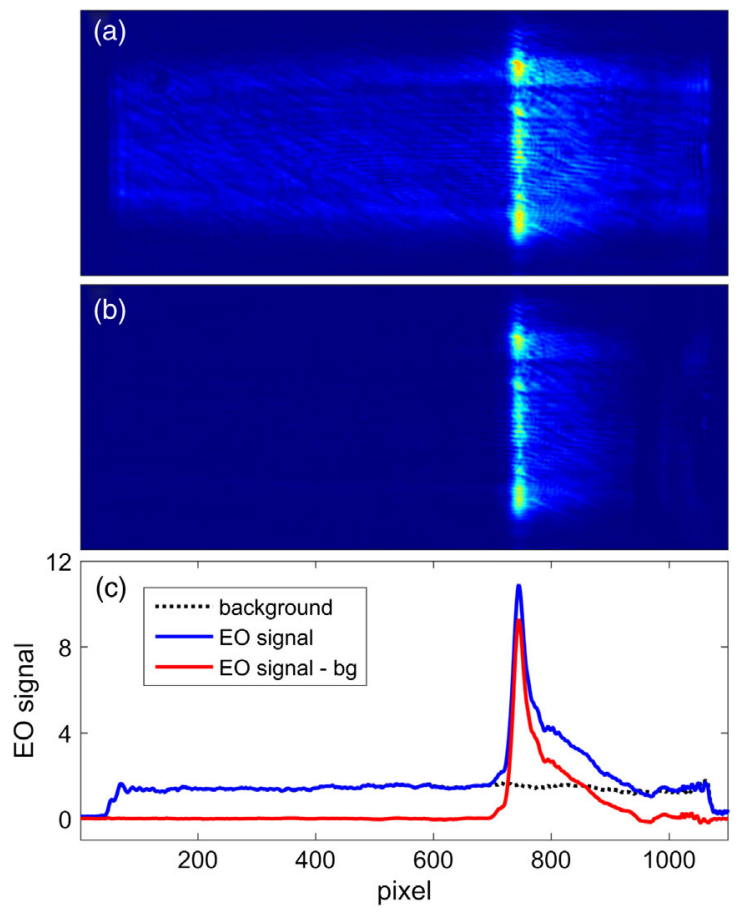

FIG. 4. Measurements of the single-shot spatial decoding EO detection at crossed polarization with $300 \mu \mathrm{m}$ ZnTe. (a) Typical raw CCD image of the probe laser profile. The left end of the laser is stopped by the crystal mounting structure, and the right is cut by the crystal edge. (b) Signal image with laser background subtracted. (c) The corresponding projected distribution of images (a) and (b). The projection of the laser profile without electron beam is plotted in dotted black curve. The bunch head is on the left. The EO trace shows a narrow spike followed by a long decreasing tail.

not taken into account. In all the following results, the factor of square root calculations has been accounted for and removed.

The time scale can be roughly calibrated by the geometric layout of the laser. The size of a CCD pixel $\Delta d$ is $\sim 5 \mu \mathrm{m}$. The cross angle of laser and beam is $\theta=40^{\circ}$. Calibration factor can be obtained by

$$
\Delta d \tan \theta / c=13.98 \text { fs/pixel. }
$$

An accurate method of time calibration is continuously varying the time delay between the electron bunch and probe laser. Changing the delay stage by a precise known value, the position of the EO signal in the laser profile also moves for a certain distance, which is sensitive to the arrival time jitter of the beam. Figure 5 shows the peak position of EO signal in the laser profile for different delays of the laser pulse. A linear fit is used to decrease the influence of time jitter including the fast term and the slow drift. The calibration factor of $13.65 \mathrm{fs} /$ pixel obtained from the fit to the peak position data is in a good agreement with the calculated factor of $13.98 \mathrm{fs} /$ pixel in Eq. (6). 


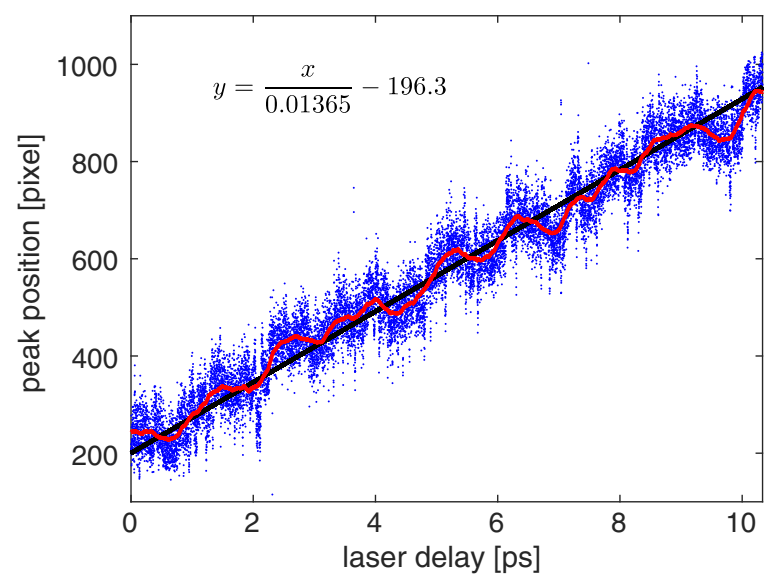

FIG. 5. Time calibration for the EO measurements. Each data point represents the peak position in the laser profile of a singleshot EO signal for different laser delays. The red curve represents the slow drift of time jitter, and the linear fit in black line provides a calibration factor of $13.65 \mathrm{fs} /$ pixel.

\section{B. Profile correction by distance}

The distance between probe laser and electron bunch is different when the head and tail of the bunch interact with laser pulse in the crystal because of the crossed incidence of the probe laser to the electron beam in the spatial decoding detection. This distance difference leads to a discrepancy of EO signal amplitude even though the Coulomb field is identical. Therefore, the measured field profile is a modulation of the bunch Coulomb field by the interaction distance. The deviation can be ignored toward ultrashort bunches for the small distance difference at the bunch head and tail. However, the observed distribution would be much different from the actual beam profile and should be corrected for a long bunch. A reasonable way is to measure EO signal amplitude of the same bunch at continuously changed distance and obtain the relationship of amplitude and distance as the correction basis seen in Fig. 6. The signal amplitude decreases rapidly with the increase of distance especially for a small distance because of the decrease of field strength. Considering the high signal-tonoise ratio, a distance as close as possible is preferred during the measurement. The amplitude $y$ can be calibrated from the distance $x$ by applying the following fit equation according to experimental data:

$$
y=\frac{1.29}{x^{2}+0.8865}-0.005923 .
$$

Note that the calculated result is the relative amplitude, which only represents the calibration factor of signal under varying distance. The amplitude is approximately inversely proportional to the square of distance for the fact that signal intensity for minimal phase retardation at crossed polarization setup is proportional to the square of field strength

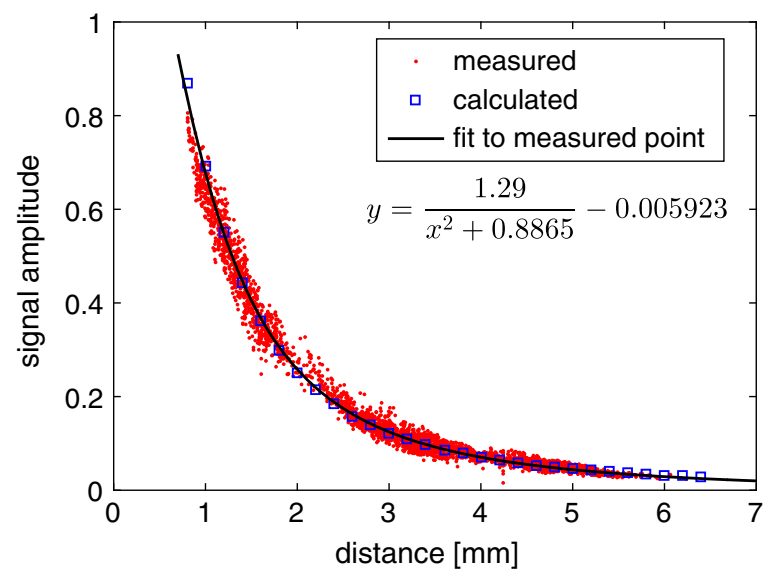

FIG. 6. EO signal amplitude of the same bunch at varying distance between laser pulse and electron beam. The amplitude of EO signal decreases rapidly with the increase of distance. The calculated data in blue square is in good agreement with the measured point. A fit according to the experimental data provides the correction basis. Continuous change of distance is realized by adjusting laser delay and taking advantage of the relative time jitter between laser pulse and electron beam.

[Eq. (5)], which is in inverse proportion to the distance for a relativistic bunch [Eq. (3)].

The EO signal trace with and without correction according to the relationship of amplitude and distance is shown in Fig. 7. The measurement is performed by $300 \mu \mathrm{m} \mathrm{ZnTe}$ for an uncompressed twin bunch at a full width of $\sim 5 \mathrm{ps}$ and total charge of $50 \mathrm{pC}$. The bunch head reaches the crystal earlier than the tail, and the probe laser is not parallel with the bunch travel direction seen in Fig. 1. Thus, the distance between the bunch head and the correspondingly interacting part of the laser is further compared with the bunch tail, leading to an amplitude reduction of the head in the EO signal. Profile distortion exists in the uncorrected data with respect to the real longitudinal bunch profile. The corrected distribution according to the fit Eq. (7) is in reasonable agreement with the TDS results. The distance of different positions within EO signal is resolved by the CCD image and geometric layout of the laser. The following results of longitudinal bunch profile have been corrected unless otherwise specified.

\section{Comparison with the transverse deflecting structure}

Single-shot spatial decoding EO measurements by $\mathrm{ZnTe}$ and $\mathrm{GaP}$ are compared with the results of deflecting cavity in Fig. 8. For the long bunch in Fig. 8(a), the three measured results are similar and demonstrate a good reconstruction at a bunch length of $\sim 1.2 \mathrm{ps}$ FWHM except for the sharp leading edge in the EO situation. With respect to $\mathrm{ZnTe}$, signal-to-noise ratio of $\mathrm{GaP}$ is low because of its thinner thickness and smaller EO coefficient. The second peak located 2.2 ps behind the main one is caused by the 


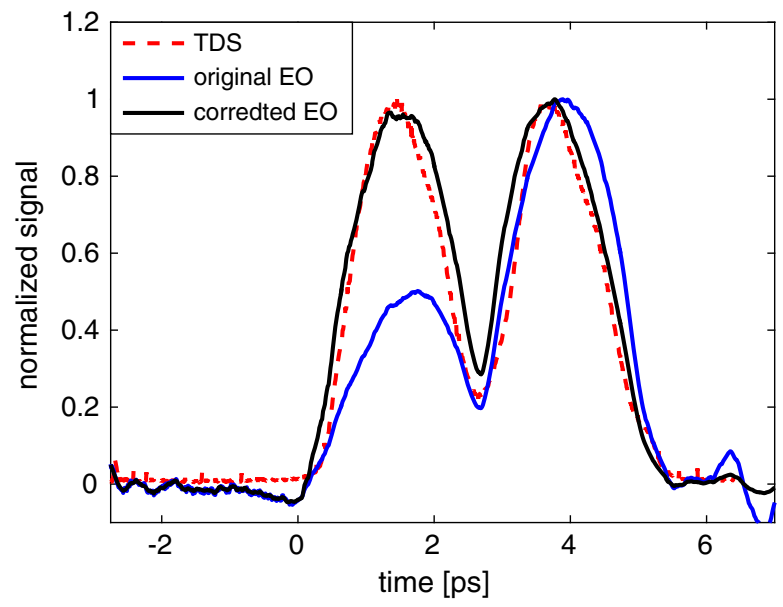

FIG. 7. Correction of spatial decoding EO measurement by distance between probe laser and electron bunch. The bunch head is on the left side. Signal-to-noise ratio is relatively low for small signal amplitude with long bunch. EO signals before and after correction are shown in blue and black lines, respectively. The TDS measurement in dotted red line is taken as the reference of the real bunch distribution.

internal reflection of Coulomb field in the GaP crystal. The reflection peak of $\mathrm{ZnTe}$ cannot be seen within the time scale, which occurs at $\sim 6$ ps behind the main peak. For short bunch with tiny inner structure in Fig. 8(b), the EO signal obtained by GaP shows a narrow peak (rms length $90 \mathrm{fs}$ ) and an obvious shoulder. The ZnTe peak is slightly broadened to an rms length of $105 \mathrm{fs}$ and covers part of the shoulder. As for the deflecting cavity in the TTX beamline, it is unable to discriminate the internal structure because of
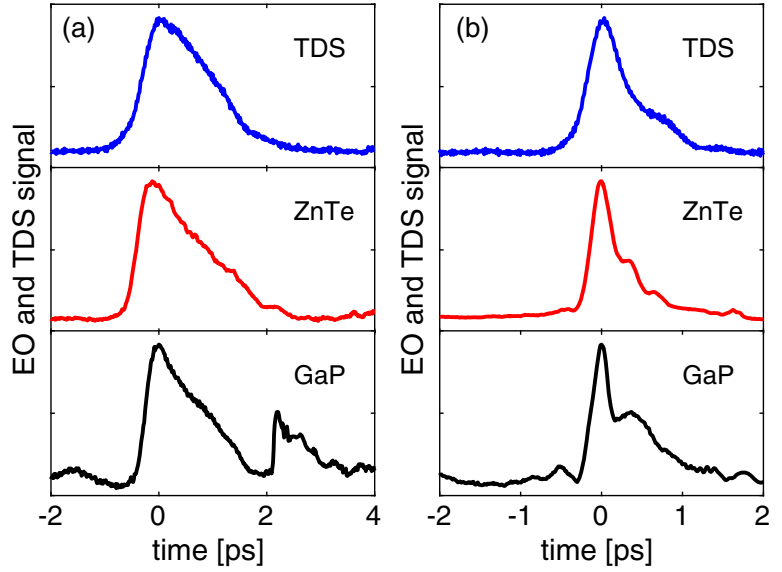

FIG. 8. Measurements of two different bunch longitudinal profiles by transverse deflecting structure (TDS) and spatial decoding EO detection with $300 \mu \mathrm{m}$ ZnTe and $100 \mu \mathrm{m}$ GaP. Three measured results under the same accelerator conditions for long bunches (a) and short bunches with tiny inner structure (b). The bunch head is on the left. At the bottom of the drawing (a), the second peak located at $2.2 \mathrm{ps}$ behind the main one is caused by the internal reflection of $\mathrm{THz}$ pulse in the GaP crystal. its poor resolution and presents only a single peak at an rms width of $220 \mathrm{fs}$.

\section{Limitation of temporal resolution}

The temporal resolution is mainly limited by the width of the probe laser, the resolution of image system, the range of the effective response bandwidth of the EO crystal, the beam energy together with the distance between electron beam and probe laser at the EO crystal, which leads to temporal broadening of Coulomb field with respect to bunch charge distribution.

A twin bunch is used to measure rf-to-laser jitter [27], and generate $\mathrm{THz}$ radiation and dual-energy X-ray at TTX. The measurement of the overcompressed twin bunch shows the shortest observed bunch profile under the current experimental conditions seen in Fig. 9. EO signals as short as $82 \mathrm{fs}$ (rms) have been observed with $100 \mu \mathrm{m} \mathrm{GaP}$. The length obtained by $300 \mu \mathrm{m} \mathrm{ZnTe}$ to the same bunch is $106 \mathrm{fs}$ (rms).

The width of probe laser is $40 \mathrm{fs}$ (FWHM), and the imaging resolution is optimized to approximately $15 \mathrm{fs}$. $\mathrm{GaP}$ crystal at a thickness of $100 \mu \mathrm{m}$ can detect electric field component with frequency up to $7.5 \mathrm{THz}$ corresponding to a minimum signal width of $\sim 60 \mathrm{fs}$ rms [28-30]. The discriminable signal width for $300 \mu \mathrm{m} \mathrm{ZnTe}$ is $\sim 110 \mathrm{fs}$ limited by its effective response frequency up to $3.75 \mathrm{THz}$ [31,32]. Resolution limits caused by the broadening of Coulomb field can be described by $\Delta t=2 r / \gamma c=80 \mathrm{fs}$ [33], where $r$ (typically $1 \mathrm{~mm}$ ) is the distance between electron bunch and probe laser, $\gamma$ is the Lorentz factor $(\gamma=80)$, and $c$ is the speed of light in vacuum.

Therefore, the measurement reaches the resolution limits of $300 \mu \mathrm{m} \mathrm{ZnTe}$, and the major limit for the measurable

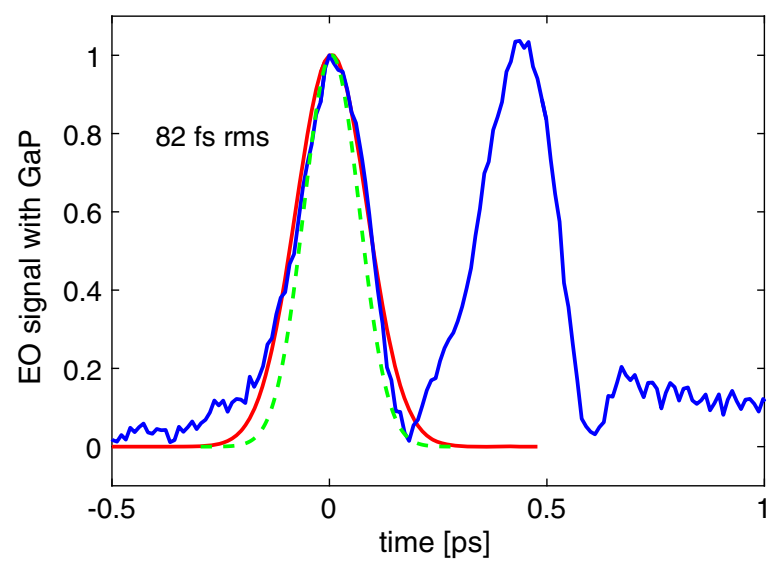

FIG. 9. Single-shot spatial decoding EO measurement of overcompressed twin bunch with $100 \mu \mathrm{m}$ GaP. Bunch head is on the left. A Gaussian fit to the front bunch is shown in red line at an rms length of $82 \mathrm{fs}$. For GaP, broadening of Coulomb field is the main reason of resolution limitation, and an rms bunch duration of $64 \mathrm{fs}$ is obtained by a deconvolution shown in green dashed line. 
bunch duration is the crystal property, i.e., the cutoff of high-frequency component of Coulomb field. A feasible way to improve the time resolution is reducing the crystal thickness to obtain high response frequency cutoff, which would also decrease the signal amplitude.

In $\mathrm{GaP}$ situation, the main limitation for the shortest measured EO signal is the widening of Coulomb field compared with bunch duration, especially for electron beam with low energy. The broadening is proportional to the field-opening angle and the distance, which cannot be too close for the nondestructive diagnostic. To remove the effect of field broadening, the charge density distribution is resolved by deconvolving single electron Coulomb field out of bunch field according to Eq. (2). The deconvolution provides a bunch width of $64 \mathrm{fs}$ rms, assuming a Gaussian distribution to the front one of the double bunch seen in Fig. 9.

\section{E. Elimination of low-energy influence}

For an electron bunch with low energy, the duration of Coulomb field increases with further distance for fieldopening angle. Therefore, the length of measured EO signal of the same bunches will change when the distance between electron bunch and probe laser is different. A varying beam arrival time relative to the laser will cause a changed distance for oblique incidence, thus a different bunch length. Figure 10 shows the measured bunch length with $300 \mu \mathrm{m}$ ZnTe and $100 \mu \mathrm{m} \mathrm{GaP}$ and the calculated Coulomb field width for different bunch lengths at varying distances.

In terms of $\mathrm{ZnTe}$, the measured bunch length reduces along with the distance decreasing, which is in agreement with the calculated curve of field duration when the distance is over $2 \mathrm{~mm}$. The bunch length tends to a fixed value of $\sim 105$ fs and remains unchanged when the distance is under $2 \mathrm{~mm}$. This tendency is caused by the confined response bandwidth and high frequency cutoff of $\mathrm{ZnTe}$, indicating that the result has reached the measurable limits. The real bunch length can be obtained by calculating the deviation between measured bunch length and field width curve with different lengths. The position of minimum deviation provides a bunch length of $61 \mathrm{fs}$ rms, plotted in bold blue line. Note that only measured data with distance above $2 \mathrm{~mm}$ is considered in the calculation of deviation for $\mathrm{ZnTe}$. Therefore, the broadening effect of field-opening angle is removed, and the monitor can be used to measure bunches with low energy. In addition, the acquired length of $61 \mathrm{fs}$ by scanning the distance is much shorter than the directly measured shortest result of 105 fs. Measuring short bunches with length of several $\sim 10$ fs can be possible by characterizing the bunch field width at different distances. The distance scanning can be simply realized by changing the laser delay in spatial decoding detection for the oblique incidence.

In $\mathrm{GaP}$, due to the small signal amplitude, the results display a large length jitter and statistics error and a narrow
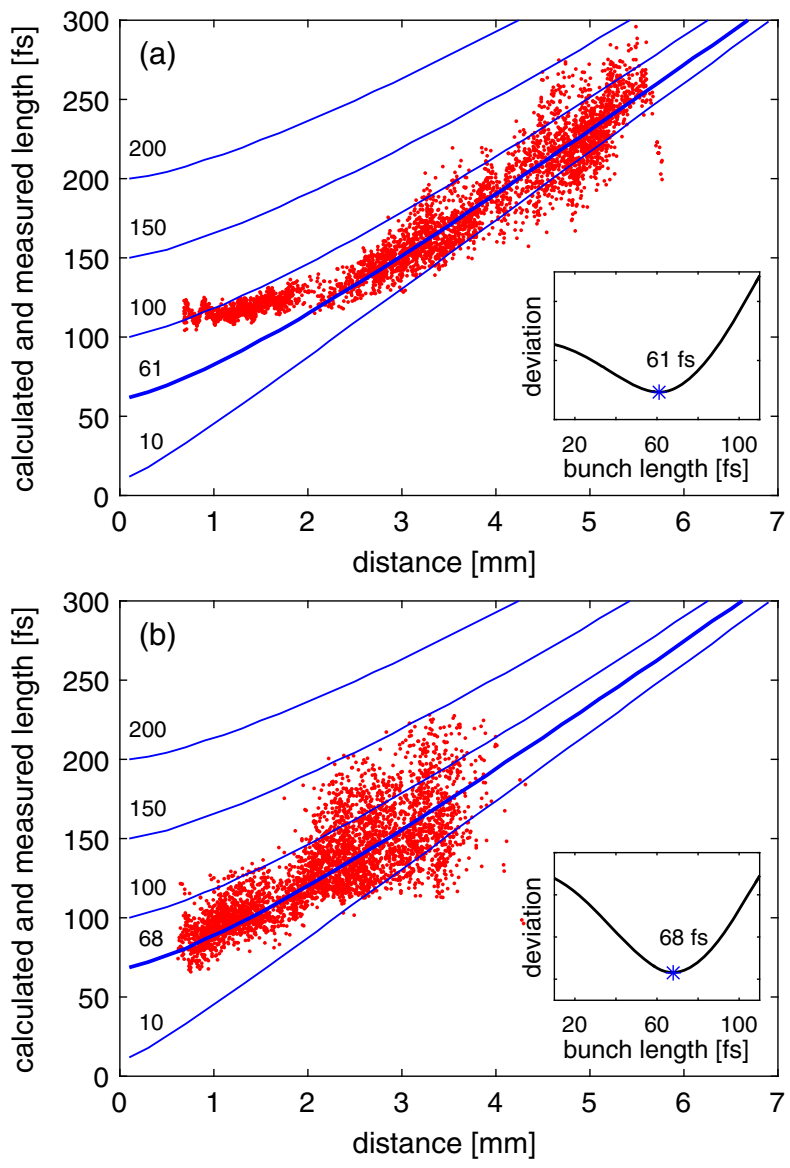

FIG. 10. Measured bunch length (red point) for the same bunches at varying distance with $300 \mu \mathrm{m} \mathrm{ZnTe} \mathrm{(a)} \mathrm{and}$ $100 \mu \mathrm{m} \mathrm{GaP}$ (b) and the calculated curve of Coulomb field width (blue line) with distance for different bunch lengths. Inset: deviation between measured results and calculated curve of different bunch lengths. According to the minimum deviation, the obtained bunch length is $61 \mathrm{fs}$ and $68 \mathrm{fs}$ for ZnTe and GaP, respectively. The large length jitter found at a far distance is mainly caused by the small signal amplitude. Note that measured data with distance under $2 \mathrm{~mm}$ is not considered in the deviation calculation for $\mathrm{ZnTe}$.

scanning range of distance compared with ZnTe. The tendency of the measured bunch length is consistent with the calculated curve in the entire measuring range of distance. The electron bunch does not exceed the measurable limitation of GaP because of its high cutoff frequency, and thus no asymptotic value is found at a small distance. An rms bunch length of $68 \mathrm{fs}$ is obtained from the statistics of deviation to remove the influence of field broadening caused by the low bunch energy. Both results of ZnTe (61 fs) and $\mathrm{GaP}(68 \mathrm{fs})$ are in reasonable agreement with the deconvolution one of 64 fs seen in Fig. 9.

\section{F. Bunch profile monitor}

As an online measuring method, the monitor has been applied to record the longitudinal profile of optimized 

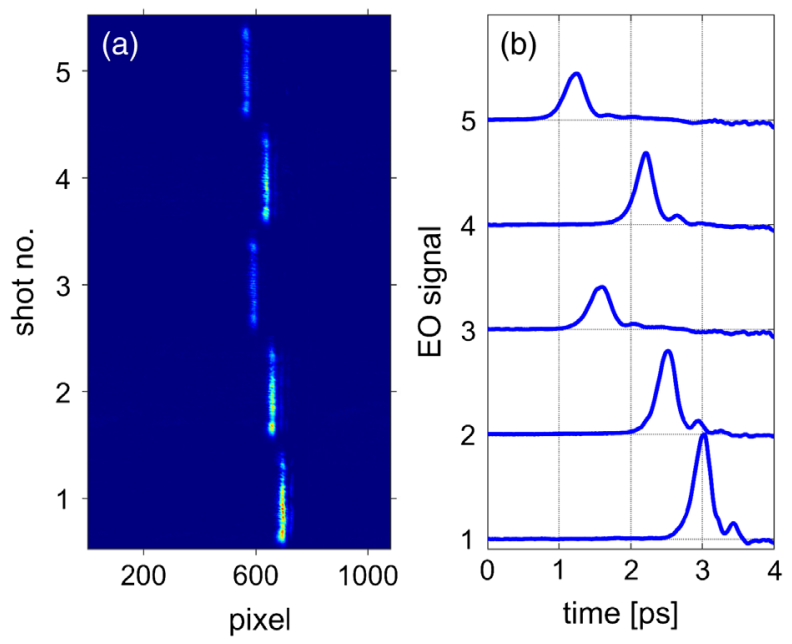

FIG. 11. Single-shot EO measurements of five consecutive electron bunches. (a) EO signal on the laser profile (background subtracted). Each bright section represents the signal, and its width and location indicate the bunch duration and relative arrival time, respectively. (b) Uncorrected longitudinal bunch profile delivered from the projection of the corresponding signal. The arrival time is defined as the time of the peak position determined by a Gaussian fit to the main peak.

compressed twin bunch. Figure 11 shows the original laser profile of EO signal and the corresponding trace of five consecutive bunches. The intensity of measured signal is mainly decided by the field strength of the bunch and the arrival time relative to the laser which determines the interaction distance. The corrected bunch profile is presented in Fig. 12 according to Sec. IV B, and the influence of distance to the signal intensity is removed. The directly
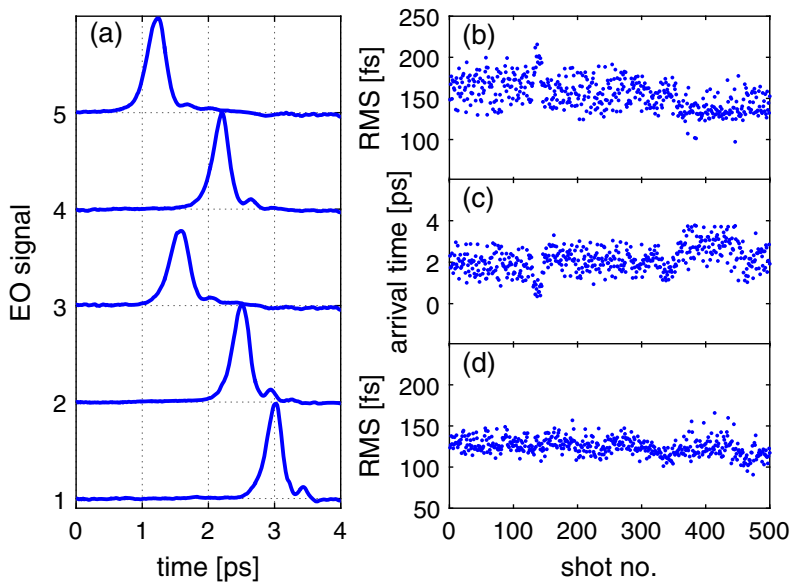

FIG. 12. Online bunch profile monitor. (a) Corrected longitudinal bunch profile of EO signal in Fig. 11 according to Eq. (7). (b) Directly measured bunch length of 500 consecutive shots. (c) Arrival time corresponding to the distance required in the following length calibration. An early arrival time means a far distance in the experimental setup. (d) Real bunch length obtained by the method in Sec. IV E. measured bunch length of 500 shots is $154 \pm 19$ fs (rms). The real bunch length is $125 \pm 10 \mathrm{fs}$ with elimination of field-opening angle according to Sec. IVE. The length deviation of the monitor caused by the arrival time jitter is also removed.

\section{G. Arrival time measurements}

Aside from the measurement of bunch profile, singleshot EO detection can simultaneously obtain the beam arrival time. The arrival time discussed here is the time jitter of the electron bunch relative to the probe laser, which would limit the time resolution of most multiple-shots pump-probe experiments [34] and degrade the stability of photon yield at Thomson scattering $\mathrm{x}$-ray source. Other experiments relying on the time synchronization of laser and bunch also need to reduce the time jitter to obtain good results.

The EO monitor has been used to measure the arrival time jitter, identify the fluctuation source, and compensate the slow drift by feedback control. The single-shot EO signal of five consecutive bunches is shown in Fig. 11. The beam arrival time jitter with respect to the laser pulse is presented as the variation of signal location in the laser profile. The arrival time defined here is the time of peak amplitude, which can be accurately determined by a Gaussian fit to the main peak excluding the slowly decaying tail of the bunch. The accuracy is approximately $10 \mathrm{fs}$ in the current experimental layout of spatially resolved measurements.

The arrival time measured over an hour during normal accelerator operation is shown in Fig. 13(a). The arrival time jitter is $430 \mathrm{fs}$ rms including the fast term and slow drift. The fast-term component or the random shot-to-shot fluctuation is $270 \mathrm{fs}$ rms and various sources are observed contributing to it. The first one is the synchronization jitter of the two laser systems to the $2856 \mathrm{MHz}$ master reference signal. The jitter of the UV drive laser and IR probe laser to this reference rf are approximately 100 and $200 \mathrm{fs}$, respectively. Another source is the instability of accelerator parameters, especially the fluctuations of $\mathrm{rf}$ phase and amplitude. Figure 13(b) shows the frequency spectrum of the arrival time jitter. The low frequency component represents the slow drift, which is generally considered to be associated with environmental temperature. The uniform noise in the high-frequency region reflects the random fast-term jitter. The obviously observed period of slow drift is $12.9 \mathrm{~min}$ corresponding to the frequency of the maximum peak (peak $3, f_{3}=$ $0.00129 \mathrm{~Hz}$ ) in the spectrum. The following five peaks represent other frequency components, and the peak frequencies are $f_{1}=0.000495 \mathrm{~Hz}, f_{2}=0.000908 \mathrm{~Hz}$, $f_{4}=0.00252 \mathrm{~Hz}, f_{5}=0.00186 \mathrm{~Hz}$, and $f_{6}=0.00309 \mathrm{~Hz}$, respectively.

The temperature and humidity in the laser room were simultaneously recorded in a step of $2 \mathrm{~s}$, seen in Fig. 14. 

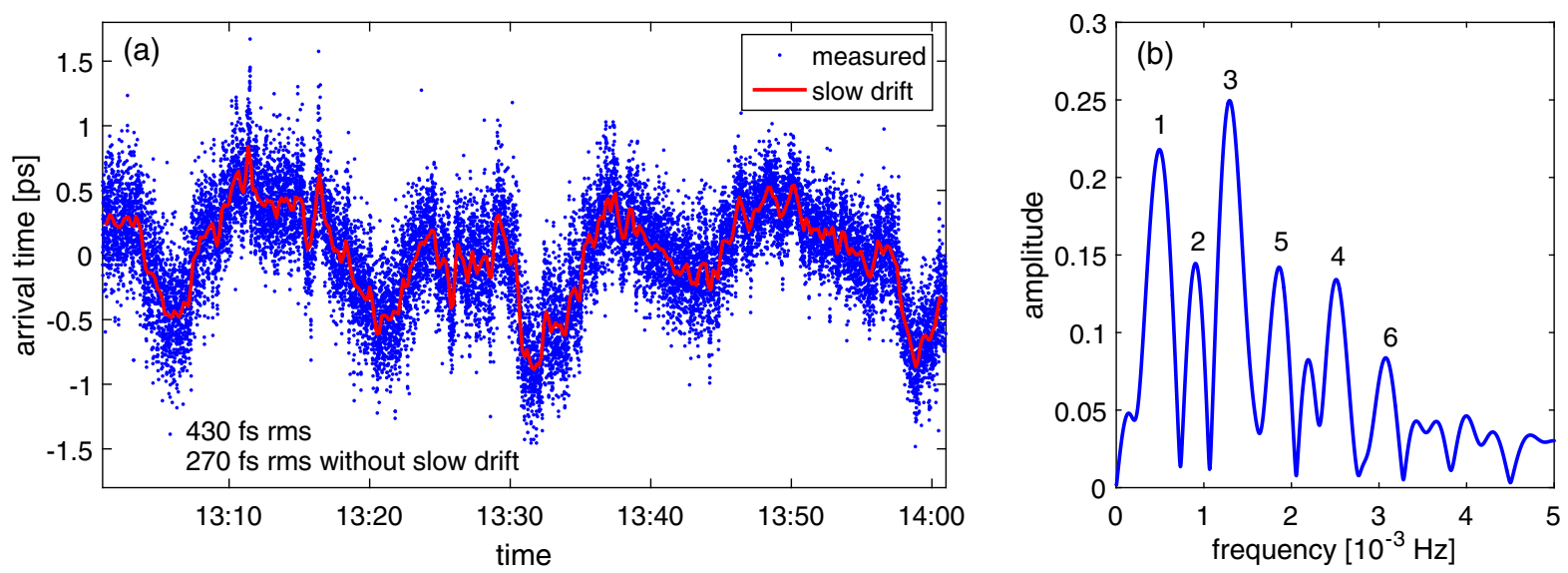

FIG. 13. (a) Beam arrival time measured in the EO experiments over an hour. The slow drift is shown in solid red line. A time jitter of $430 \mathrm{fs}$ rms is found from the data. The resulting fast-term jitter is $270 \mathrm{fs}$ rms without slow drift. (b) Frequency spectrum of the data point by Fourier transform in the low-frequency region. The frequencies of the five peaks are $f_{1}=0.000495 \mathrm{~Hz}, f_{2}=0.000908 \mathrm{~Hz}$, $f_{3}=0.00129 \mathrm{~Hz}, f_{4}=0.00252 \mathrm{~Hz}, f_{5}=0.00186 \mathrm{~Hz}$, and $f_{6}=0.00309 \mathrm{~Hz}$.

The temperature is controlled within $22.5 \pm 0.7^{\circ} \mathrm{C}$ by the air conditioner and varies in a period of 13.2 min corresponding to the frequency of peak $3\left(f_{3}=0.00126 \mathrm{~Hz}\right)$. The humidity ranges from $18 \%$ to $36 \%$, and the main period is $40.2 \mathrm{~min}$ resulting from the frequency of peak 1 $\left(f_{1}=0.000415 \mathrm{~Hz}\right)$. The rest of relatively obvious peaks are peak 2 of humidity and peak 4 of temperature at a frequency of $f_{2}=0.000844 \mathrm{~Hz}$ and $f_{4}=0.00251 \mathrm{~Hz}$. In the low-frequency region, the frequency components of arrival time from peaks 1 to 4 are in good agreement with the Fourier spectrum of the temperature and humidity in the laser room. Thus, the slow drift of arrival time is proved to be mainly caused by the environmental conditions, leading to periodical change of laser system and timing synchronization (placed in laser room currently) between oscillator and reference rf signal. Other components of the arrival time slow drift, such as peaks 5 and 6 in Fig. 13(b), are probably caused by the temperature variation of the cooling water to the gun and accelerator section, or even the experimental hall.

To remove the slow drift, an optional way is to update the air conditioner to improve the experimental environmental conditions, leading to high costs. Based on the arrival time information from the EO monitor, a verification experiment is performed to remove the slow drift of the arrival time with feedback control by adjusting the gun phase instead. The average of initial 100 shots is considered as the reference data, and gun phase is reset after each 50 shots according to the difference between the average of the latest 50 shots and the reference basis. Beam arrival time measurements with feedback over an hour are shown in Fig. 15.

The time jitter is reduced to $320 \mathrm{fs}$ rms (430 fs rms without EO feedback). The slow drift is basically removed,
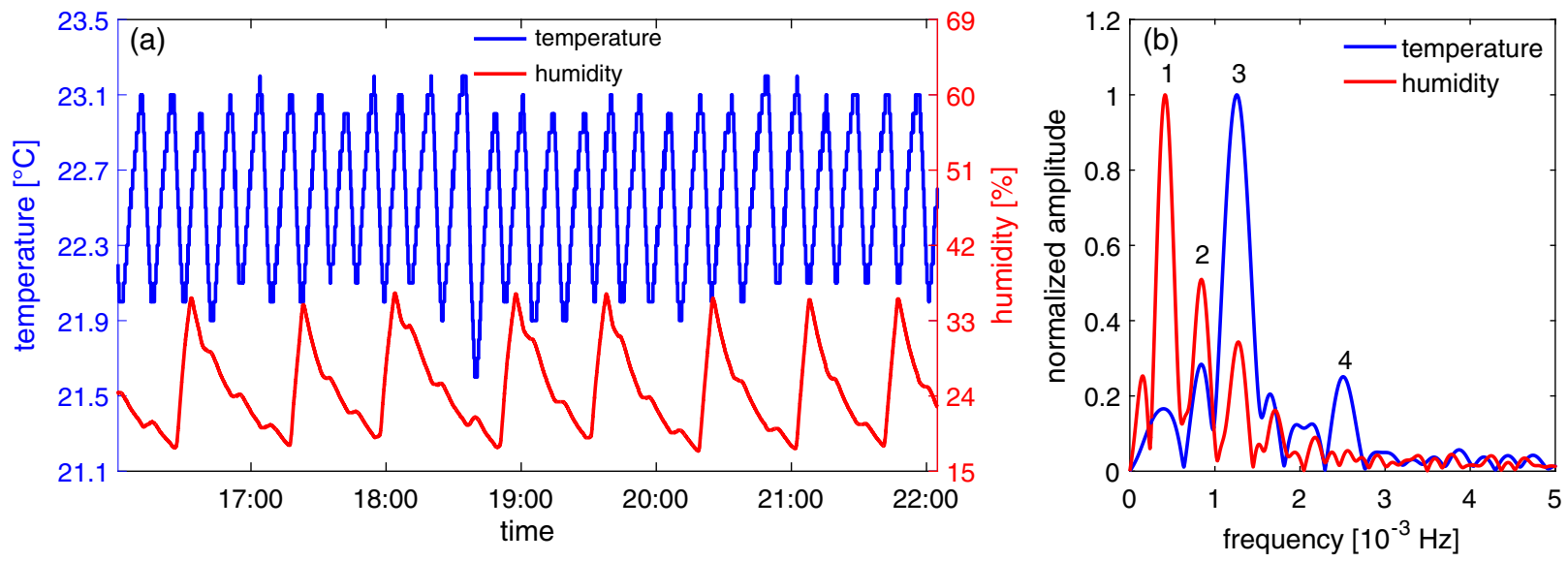

FIG. 14. Temperature and humidity in the laser room and their Fourier transform. (a) In the long-time recording, temperature and humidity show a period of $\sim 13.2$ and $\sim 40.2 \mathrm{~min}$, respectively. (b) The frequencies of the four peaks are $f_{1}=0.000415 \mathrm{~Hz}$, $f_{2}=0.000844 \mathrm{~Hz}, f_{3}=0.00126 \mathrm{~Hz}$, and $f_{4}=0.00251 \mathrm{~Hz}$. 

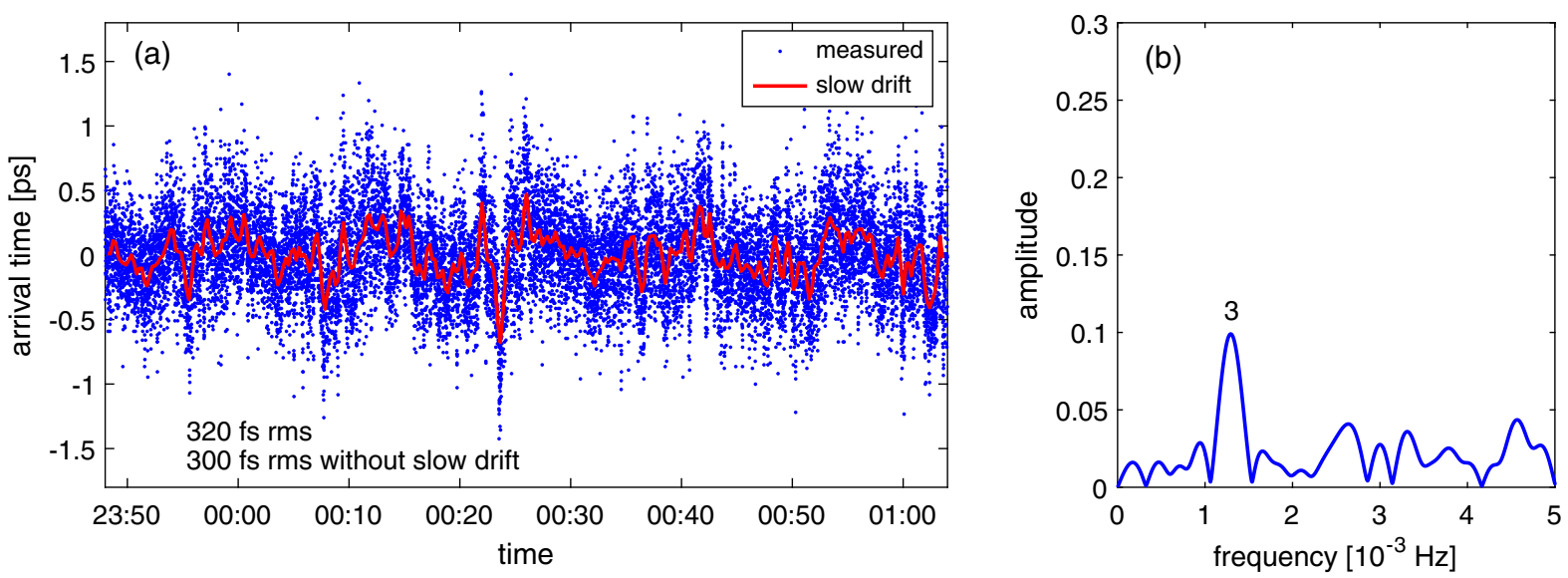

FIG. 15. (a) Beam arrival time measured with feedback control in the EO experiments over an hour. The slow drift is shown in solid red line. The rms time jitter is decreased to $320 \mathrm{fs}$. The corresponding fast-term jitter without slow drift is, however, increased to $300 \mathrm{fs}$ rms. (b) Frequency spectrum of arrival time by Fourier transform in the low frequency region. Vertical axis is set in the same scale with Fig. 13(b) for comparison. The peak frequency is $f_{3}=0.00129 \mathrm{~Hz}$.

and the low-frequency component in the spectrum is degraded to the level of high-frequency noise. The residual slow drift (peak 3) is not completely cleared because of the hysteresis of feedback control. The fastterm component of time jitter slightly rises to $300 \mathrm{fs}$ rms compared with the result of $270 \mathrm{fs}$ when the feedback is off. We attribute the increase to the way of feedback by changing the electron gun phase, which would raise the shot-to-shot fluctuation. Figure 16 shows the gun phase during the feedback process. The phase is set at $30^{\circ}$ initially and ranges from $27^{\circ}$ to $31^{\circ}$. The gun phase shows the same alternating process except for a time delay to the slow drift of arrival time. The setting of gun phase is a proof to feasibility, and a practical way to compensate the slow drift is adjusting the delay time stage of the probe laser to avoid the increase of fast-term jitter. By improving the feedback procedure, arrival time jitter of $280 \mathrm{fs}$ rms could be achieved.

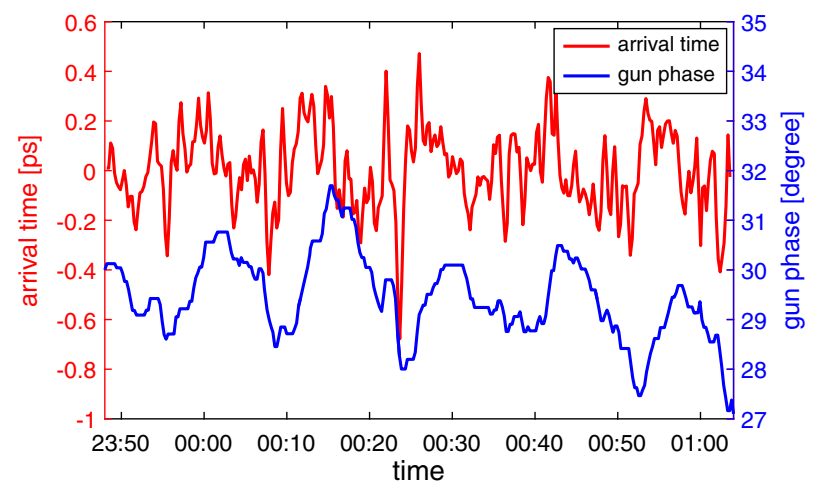

FIG. 16. Adjustment of the rf gun phase (blue line) and slow drift (red line) during the feedback process. The initial phase is $30^{\circ}$, and the variation range is approximately $4^{\circ}$.

\section{CONCLUSION}

In summary, a monitor based on spatial decoding EO detection has been successfully implemented to measure bunch temporal profile and beam arrival time in a singleshot and nondestructive way. This kind of monitor shows better time resolution than the transverse deflecting cavity at TTX. To overcome the intrinsic disadvantage of spatial decoding for oblique incidence, a calibration formula is acquired for bunch profile correction to remove the distance modulation especially for a long bunch.

The current resolution limit for $\mathrm{ZnTe}$ is the response bandwidth of the crystal, and a bunch length of $106 \mathrm{fs}$ (rms) is received. In terms of $\mathrm{GaP}$, the field-opening angle of a $40 \mathrm{MeV}$ low-energy bunch limits the measurable shortest EO signal of $82 \mathrm{fs}$ (rms). By measuring and analyzing the relationship between field width and interaction distance, the field broadening is eliminated, and real bunch length is characterized. Thus, the monitor is able to measure bunches with low energy, and bunch length of $\sim 60 \mathrm{fs} r \mathrm{rs}$ is obtained with $300 \mu \mathrm{m}$ ZnTe.

As an extended application, the monitor is used to measure the time jitter between electron bunch and synchronized laser at an estimated time resolution of 10 fs. The measured rms jitter is $430 \mathrm{fs}$ including the fast-term component (270 fs) and slow drift (330 fs). The spectral analysis of the jitter indicates that the temperature and humidity in the laser room are the main sources of the slow drifts. A verifying experiment of feedback control decreases the jitter from 430 fs to $320 \mathrm{fs}$, and the monitor can be a powerful tool to remove the slow drift component of the time jitter.

We plan to generate low-energy bunches of several MeV and ultrashort bunches of $\sim 10$ fs to test the capability of the monitor in low-energy region and femtosecond region. An improved feedback method is also expected to obtain a 
smaller time jitter. As a further step, a compact EO monitor within a normal beam instrumentation chamber unit is under consideration to serve for the Thomson scattering, pump-probe, and other synchronization experiments.

\section{ACKNOWLEDGMENTS}

This work was supported by the National Natural Science Foundation of China (NSFC Grants No. 11475097) and the National Key Scientific Instrument and Equipment Development Project of China (Grants No. 2013YQ12034504).

[1] B. Faatz, J. Pflüger, J. Rossbach, E. Saldin, E. Schneidmiller, and M. Yurkov, Parameter optimization of X-ray freeelectron lasers at a linear collider, Nucl. Instrum. Methods Phys. Res., Sect. A 407, 302 (1998).

[2] L. Bentson, P. Bolton, E. Bong, P. Emma, J. Galayda, J. Hastings, P. Krejcik, C. Rago, J. Rifkin, and C. Spencer, FEL research and development at the SLAC subpicosecond photon source, SPPS, Nucl. Instrum. Methods Phys. Res., Sect. A 507, 205 (2003).

[3] M. Altarelli, R. Brinkmann, M. Chergui, W. Decking, B. Dobson, S. Düsterer, G. Grübel, W. Graeff, H. Graafsma, J. Hajdu et al., Technical design report, DESY 97, 1 (2006).

[4] M. Hentschel, R. Kienberger, C. Spielmann, G. A. Reider, N. Milosevic, T. Brabec, P. Corkum, U. Heinzmann, M. Drescher, and F. Krausz, Attosecond metrology, Nature (London) 414, 509 (2001).

[5] X. J. Wang, D. Xiang, T. K. Kim, and H. Ihee, Potential of femtosecond electron diffraction using near-relativistic electrons from a photocathode RF electron gun, J. Korean Phys. Soc. 48, 390 (2006).

[6] A. Azima, S. Düsterer, P. Radcliffe, H. Redlin, N. Stojanovic, W. Li, H. Schlarb, J. Feldhaus, D. Cubaynes, M. Meyer et al., Time-resolved pump-probe experiments beyond the jitter limitations at FLASH, Appl. Phys. Lett. 94, 144102 (2009).

[7] C. Scoby, P. Musumeci, J. Moody, and M. Gutierrez, Electro-optic sampling at 90 degree interaction geometry for time-of-arrival stamping of ultrafast relativistic electron diffraction, Phys. Rev. ST Accel. Beams 13, 022801 (2010).

[8] I. Wilke, A. M. MacLeod, W. A. Gillespie, G. Berden, G. Knippels, and A. Van Der Meer, Single-Shot ElectronBeam Bunch Length Measurements, Phys. Rev. Lett. 88, 124801 (2002).

[9] G. Berden, S. P. Jamison, A. M. MacLeod, W. A. Gillespie, B. Redlich, and A. van der Meer, Electro-Optic Technique with Improved Time Resolution for Real-Time, Nondestructive, Single-Shot Measurements of Femtosecond Electron Bunch Profiles, Phys. Rev. Lett. 93, 114802 (2004).

[10] A. L. Cavalieri, D. Fritz, S. Lee, P. Bucksbaum, D. Reis, J. Rudati, D. Mills, P. Fuoss, G. Stephenson, C. Kao et al., Clocking Femtosecond X Rays, Phys. Rev. Lett. 94, 114801 (2005).

[11] G. Berden, W. A. Gillespie, S. P. Jamison, E.-A. Knabbe, A. M. MacLeod, A. van der Meer, P. J. Phillips, H. Schlarb,
B. Schmidt, P. Schmüser et al., Benchmarking of ElectroOptic Monitors for Femtosecond Electron Bunches, Phys. Rev. Lett. 99, 164801 (2007).

[12] X. Yang, T. Tsang, T. Rao, J. Murphy, Y. Shen, and X. Wang, Electron bunch length monitors using spatially encoded electro-optical technique in an orthogonal configuration, Appl. Phys. Lett. 95, 231106 (2009).

[13] D. Sütterlin, D. Erni, V. Schlott, H. Sigg, H. Jäckel, and A. Murk, Single-shot electron bunch length measurements using a spatial electro-optical autocorrelation interferometer, Rev. Sci. Instrum. 81, 104702 (2010).

[14] B. Steffen, V. Arsov, G. Berden, W. A. Gillespie, S. Jamison, A. M. MacLeod, A. van der Meer, P. Phillips, H. Schlarb, B. Schmidt et al., Electro-optic time profile monitors for femtosecond electron bunches at the soft x-ray free-electron laser FLASH, Phys. Rev. ST Accel. Beams 12, 032802 (2009).

[15] M. H. Helle, D. F. Gordon, D. Kaganovich, and A. Ting, Extending electro-optic detection to ultrashort electron beams, Phys. Rev. ST Accel. Beams 15, 052801 (2012).

[16] F. Müller, P. Peier, V. Schlott, B. Steffen, T. Feurer, and P. Kuske, Electro-optical measurement of sub-ps structures in low charge electron bunches, Phys. Rev. ST Accel. Beams 15, 070701 (2012).

[17] J. Van Tilborg, C. Schroeder, C. Filip, C. Tóth, C. Geddes, G. Fubiani, R. Huber, R. Kaindl, E. Esarey, and W. Leemans, Temporal Characterization of Femtosecond Laser-Plasma-Accelerated Electron Bunches Using Terahertz Radiation, Phys. Rev. Lett. 96, 014801 (2006).

[18] A. Debus, M. Bussmann, U. Schramm, R. Sauerbrey, C. Murphy, Z. Major, R. Hörlein, L. Veisz, K. Schmid, J. Schreiber et al., Electron Bunch Length Measurements from Laser-Accelerated Electrons Using Single-Shot THz Time-Domain Interferometry, Phys. Rev. Lett. 104, 084802 (2010).

[19] J.D. Jackson, Electrodynamics (Wiley Online Library, New York, 1975).

[20] S. Casalbuoni, H. Schlarb, B. Schmidt, B. Steffen, P. Schmuser, and A. Winter, Numerical studies on the electrooptic sampling of relativistic electron bunches, in Proceedings of the 21st Particle Accelerator Conference, Knoxville, TN, 2005 (IEEE, Piscataway, NJ, 2005), p. 3070.

[21] S. Casalbuoni, H. Schlarb, B. Schmidt, P. Schmüser, B. Steffen, and A. Winter, Numerical studies on the electrooptic detection of femtosecond electron bunches, Phys. Rev. ST Accel. Beams 11, 072802 (2008).

[22] B. Steffen, Ph.D. thesis, University of Hamburg, Hamburg, Germany, 2007, Report No. DESYTHESIS-2007-020.

[23] Y. Du, L. Yan, J. Hua, Q. Du, Z. Zhang et al., Generation of first hard X-ray pulse at Tsinghua Thomson Scattering X-ray Source, Rev. Sci. Instrum. 84, 053301 (2013).

[24] Z. Chi, L. Yan, Y. Du, Z. Zhang, W. Huang, H. Chen, and C. Tang, Recent progress of phase-contrast imaging at Tsinghua Thomson-scattering X-ray source, Nucl. Instrum. Methods Phys. Res., Sect. B 402, 364 (2017).

[25] L. Yan, Q. Du, Y. Du, J. Hua, W. Huang, and C. Tang, UV pulse shaping for the photocathode RF gun, Nucl. Instrum. Methods Phys. Res., Sect. A 637, S127 (2011).

[26] L.-X. Yan, J.-F. Hua, Y.-C. Du, Y.-F. Huang, Y. You, D. Wang, W.-H. Huang, and C.-X. Tang, UV pulse trains by 
$\alpha$-BBO crystal stacking for the production of THz-rap-rate electron bunches, J. Plasma Phys. 78, 429 (2012).

[27] Z. Zhang, Y. Du, L. Yan, Q. Du, J. Hua, J. Shi, J. Yang, D. Wang, W. Huang, H. Chen et al., High time resolution beam-based measurement of the rf-to-laser jitter in a photocathode rf gun, Phys. Rev. ST Accel. Beams 17, 032803 (2014).

[28] D. Kleinman and W. Spitzer, Infrared Lattice Absorption of GaP, Phys. Rev. 118, 110 (1960).

[29] Q. Wu and X.-C. Zhang, 7 terahertz broadband GaP electro-optic sensor, Appl. Phys. Lett. 70, 1784 (1997).

[30] D. Nelson and E. Turner, Electro-optic and Piezoelectric Coefficients and Refractive Index of Gallium Phosphide, J. Appl. Phys. 39, 3337 (1968).
[31] T. Sliker and J. Jost, Linear Electro-Optic Effect and Refractive Indices of Cubic ZnTe, J. Opt. Soc. Am. 56, 130 (1966).

[32] S. Casalbuoni et al., Tesla Report No. 2005-01, 2005.

[33] X. Yan, A. MacLeod, W. Gillespie, G. Knippels, D. Oepts, A. van der Meer, and W. Seidel, Subpicosecond Electrooptic Measurement of Relativistic Electron Pulses, Phys. Rev. Lett. 85, 3404 (2000).

[34] S. Weathersby, G. Brown, M. Centurion, T. Chase, R. Coffee, J. Corbett, J. Eichner, J. Frisch, A. Fry, M. Gühr et al., Mega-electron-volt ultrafast electron diffraction at SLAC National Accelerator Laboratory, Rev. Sci. Instrum. 86, 073702 (2015). 